PREPARED FOR THE U.S. DEPARTMENT OF ENERGY, UNDER CONTRACT DE-AC02-76CH03073

PPPL-3480

PPPL-3480

UC-70

APD Detector Electronics for the NSTX Thomson Scattering System

by

D.W. Johnson, B.P. LeBlanc, D.L. Long, and G. Renda

August 2000

$=$ PPPL

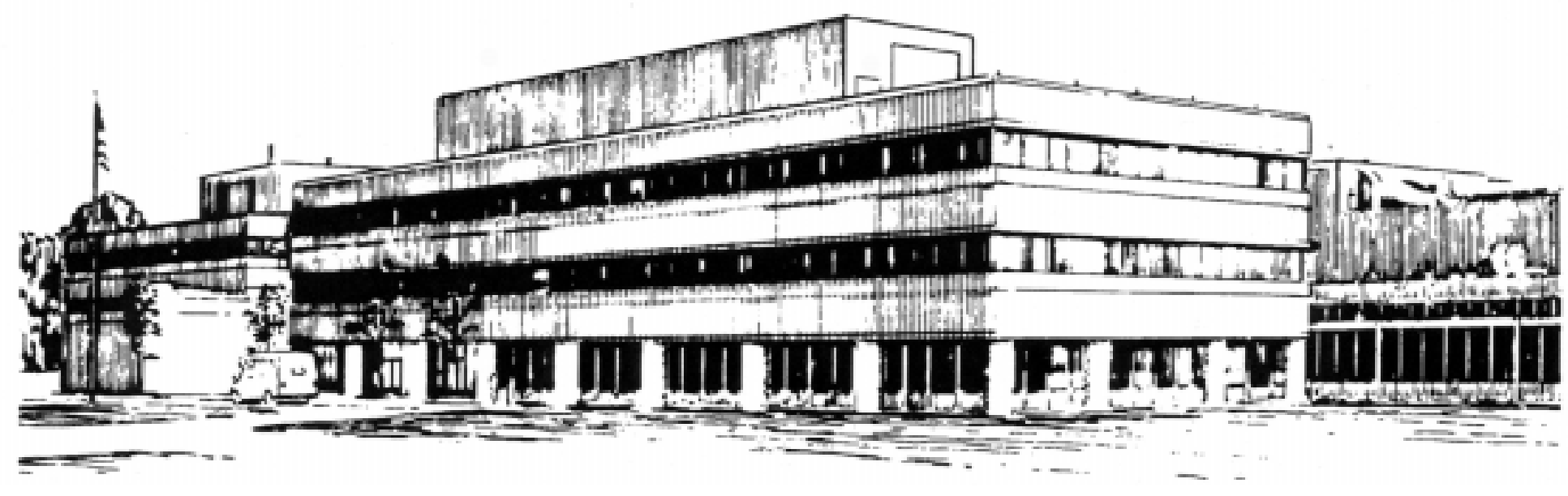

PRINCETON PLASMA PHYSICS LABORATORY PRINCETON UNIVERSITY, PRINCETON, NEW JERSEY 


\section{PPPL Reports Disclaimer}

This report was prepared as an account of work sponsored by an agency of the United States Government. Neither the United States Government nor any agency thereof, nor any of their employees, makes any warranty, express or implied, or assumes any legal liability or responsibility for the accuracy, completeness, or usefulness of any information, apparatus, product, or process disclosed, or represents that its use would not infringe privately owned rights. Reference herein to any specific commercial product, process, or service by trade name, trademark, manufacturer, or otherwise, does not necessarily constitute or imply its endorsement, recommendation, or favoring by the United States Government or any agency thereof. The views and opinions of authors expressed herein do not necessarily state or reflect those of the United States Government or any agency thereof.

\section{Availability}

This report is posted on the U.S. Department of Energy's Princeton Plasma Physics Laboratory Publications and Reports web site in Calendar Year 2000. The home page for PPPL Reports and Publications is: http://www.pppl.gov/pub_report/

DOE and DOE Contractors can obtain copies of this report from:

U.S. Department of Energy

Office of Scientific and Technical Information

DOE Technical Information Services (DTIS)

P.O. Box 62

Oak Ridge, TN 37831

Telephone: (865) 576-8401

Fax: (865) 576-5728

Email: reports@adonis.osti.gov

This report is available to the general public from:

National Technical Information Service

U.S. Department of Commerce

5285 Port Royal Road

Springfield, VA 22161

Telephone: $1-800-553-6847$ or

(703) $605-6000$

Fax: (703) 321-8547

Internet: http://www.ntis.gov/ordering.htm 


\title{
APD detector electronics for the NSTX Thomson scattering system
}

D.W. Johnson, B.P. LeBlanc, D.L. Long, and G. Renda*

Princeton Plasma Physics Laboratory, Princeton, New Jersey, 08543

*Princeton Scientific Instruments, Princeton, New Jersey, 08544

\begin{abstract}
An electronics system has been installed and tested for the readout of APD detectors for the NSTX Thomson scattering system. Similar to previous designs, it features preamps with a fast and a slow output. The fast output uses pulse shaping to optimize sensitivity for the 8 nsec scattered light pulse while rejecting noise in the intrinsic plasma background. A low readout noise of $\sim 25$ photoelectrons is achieved at an APD gain of 75 . The design incorporates a number of features to provide flexibility for various modes of calibration.
\end{abstract}

\section{INTRODUCTION}

Avalanche photodiodes (APDs) have been used as detectors for a number of Thomson scattering systems employing Nd:YAG lasers at $1.06 \mu \mathrm{m} .{ }^{1-5}$ A similar system has recently been installed on $\mathrm{NSTX}^{6}$, with initial operation scheduled for July, 2000. This paper describes the design and lab performance of this electronics.

The detectors are EG\&G APD type C30956E. They have a $3 \mathrm{~mm}$ diameter with a quantum efficiency of $70-80 \%$ from $750 \rightarrow 950 \mathrm{~nm}$ falling to $30-40 \%$ at $1050 \mathrm{~nm}$. The APDs can be operated at a gain $M$ up to 100 by setting a bias voltage up to 400 volts. At a given voltage the gain varies from device to device, and the excess noise in the device increases with gain. ${ }^{7}$ The gain also varies significantly with temperature.

Scattered light, transported from NSTX through a fiber optic bundle, illuminates the detectors through one of six interference filters in a filter polychromator, identical to that 
described in ref. 3 . The system is designed for $\sim 40$ such polychromators, which would require $\sim 240$ sets of electronics, although only 10 polychromators will be used initially.

\section{PREAMPLIFIERLIFIER MODULE}

The preamplifier electronics is designed with varied goals in mind. It has to measure intensities from $50 \rightarrow 10^{5}$ photoelectrons from a $8 \mathrm{nsec}$ pulsed source in the presence of intrinsic plasma light background with a brightness of up to $10^{12}$ photoelectrons/sec. It should maximize the rejection of noise from the background light. In order to take this noise into account in the subsequent analysis, it should also measure the plasma light, with an integration time of $\sim 1 \mu \mathrm{s}$. To provide spectral calibration using a DC light source, a clear means of relating the transfer function is needed for pulsed vs DC sources. To optimize individual detector performance, the APD gain (bias voltage) should be independently, remotely selectable with crowbar protection. To eliminate temporal DC offset drift for DC measurements, an auto-zero feature is needed to zero the output just prior to illumination.

While the APDs are available from EG\&G with low noise, integrated preamplifiers, correlating the pulsed response with the DC response of such detectors is difficult, and we chose instead to use discrete detectors.

The preamplifier was designed by Princeton Scientific Instruments. Similar to other designs, ${ }^{8,9}$ the circuit has two outputs. A slow output is used to measure the plasma background light and DC calibration sources. The fast output is optimized for measuring pulses similar to the scattered light pulse, while rejecting noise due to the plasma background light. Pulse shaping in the fast channel uses a low-pass filter with a resistor and capacitor network in the feedback loop of the input amplifier, and a high-pass section which effectively subtracts a delayed from a nondelayed signal. The preamp circuit is shown in Fig. 1. It uses a Comlinear CLC425 low noise, wideband amplifier with $\mathrm{C}^{\mathrm{FB}}=1 \mathrm{pf}$ and $\mathrm{R}^{\mathrm{FB}}=50 \mathrm{k} \Omega$ in the feedback loop. This design results in 
a photoelectron equivalent noise of $\sim 25$ photoelectrons at $M=75$. This is significantly lower than previous designs. A two-pole Bessel filter substitutes for a delay line in the subtraction circuit. The response of the fast output to a 8 nsec optical pulse is shown in Fig. 2.

In order to optimize the operating point for each APD separately, depending on individual detector characteristics and illumination conditions, each preamplifier module contains a HV supply (Analog Modules type 521-5, 0 to +500 volts ) adjustable using an analog output from a CAMAC DAC module. To protect the APD, a crowbar circuit sets the program voltage to zero if a preset HV output is exceeded. To provide first order compensation for the variation of APD gain with temperature $(\sim 2 \%$ per degree $\mathrm{C}$ uncompensated at $\mathrm{M}=75)$, the program voltage is varied slightly from the DAC value, by a circuit that senses the temperature with a diode which is thermally sinked to the same plate as the APD. The bias voltage and the program voltage are sampled and archived, to provide information on the gain and temperature.

The preamplifier module is housed with the APD in an aluminum enclosure with outer dimensions 34x100x207 mm. The APD is clamped with good thermal contact in a hole in the $6.35 \mathrm{~mm}$ thick end plate. The precision hole is justified to two locating pins which center the detector at the focus of the polychromator lens. The end plate and lens housing are thermally coupled to the side plate of the filter polychromator box, which has channels for water circulation to provide temperature stability for the filters and detectors. The lens housing also has an SMA connector and lens to image the output of an optical fiber directly onto the APD, along an axis sufficiently skewed that these elements do not obscure light coming through the interference filter. This alternate illumination port is used in detector calibrations described below. 


\section{SAMPLE AND HOLD - MULTIPLEXER MODULE}

In most existing systems, the preamplifier signals are digitized in commercial gated integrators, such as the LeCroy FERA CAMAC modules. We have chosen to build a sample and hold/multiplexer (SHMUX) module which amplifies and samples the 6 pulse outputs along with the 6 slow outputs from a polychromator and multiplexes them for input, along with a suitable clock, to a general purpose digitizer. The SHMUX output can be strapped for either $-5 \rightarrow+5 \mathrm{v}$ or $0 \rightarrow 10 \mathrm{v}$ operation. We are currently using a 12 bit digitizer. The pulse outputs are sampled with a fast sample and hold (Datel SHM-14) with a acquisition time of $10 \mathrm{nsec}$ for $0.1 \%$ accuracy on a square wave. A second sample and hold is used in series to avoid droop during the multiplexing phase, which takes $\sim 400 \mu$ s. The SHMUX modules fit into a Euro nest (16/nest) with a synchronization module that interfaces to various trigger sources and provides timing adjustment for synchronization of the fast sample with the laser. Fig. 3 shows the physical layout of the polychromators, preamps, and SHMUX nest.

\section{SYNCHRONIZATION}

In normal operation, a small amount of the Nd:YAG laser light is imaged onto an optical fiber which is sensed at the synchronizer module and used to trigger the samples. As shown in fig. 2, the window for sampling the preamplifier output peak is only a few nsec wide. Thus, to account for optical path differences of up to $\sim 12$ nsec between edges of the imaged laser path in the plasma, a means of independently synchronizing the SHMUX is needed. Each SHMUX circuit includes a time delay that can be strapped in $2 \mathrm{nsec}$ intervals over a range of $20 \mathrm{nsec}$. Also, the synchronizer module contains both a delay that can be strapped over a 5-50 nsec range, and a computer selectable delay that can be programmed in $2 \mathrm{nsec}$ steps over a range of $32 \mathrm{nsec}$. Rayleigh scattering is used to check the synchronization. Initially, cable lengths and strapped delays are set to best guess values. Rayleigh scattering data is taken while scanning through the programmable delay. Small changes to optimize are then made to the strapped delays. 


\section{TRANSFER FUNCTIONS}

The transfer functions for the fast and slow channels are very different and are clearly frequency dependent. This is important, since spectral calibrations must be done with DC sources, and so a DC photon flux must be related to spectral variations in the scattered fast pulse signals. The transfer functions can be written:

$$
\begin{aligned}
& V^{\text {fast }}=\frac{N_{p h}^{\text {fast }} Q M e G^{\text {fast }}}{C^{F B}} \approx N_{p h}^{\text {fast }} Q M\left(1 \times 10^{-6} v / \text { photoele. }\right) \\
& V^{\text {slow }}=I_{p h}^{\text {slow }} Q M e R^{F B} G^{\text {slow }} \approx I_{p h}^{\text {slow }} Q M\left(3 \times 10^{-13} v / \text { photoele./s }\right)
\end{aligned}
$$

where $\mathrm{V}$ is the output voltage, $\mathrm{N}$ is the number of integrated photons in the fast pulse, $\mathrm{I}$ is the background photon flux or the photon flux from a calibration source, $\mathrm{Q}$ is the quantum efficiency, M is the APD gain, $\mathrm{e}$ is the electronic charge, $\mathrm{G}$ is the gain of the respective chains of amplifiers after $\mathrm{U} 1$ (see fig. 1), and $\mathrm{C}^{\mathrm{FB}}$ and $\mathrm{R}^{\mathrm{FB}}$ are the components in the feedback loop of $\mathrm{U} 1$.

If we use nominal values for the gains and for $\mathrm{R}^{\mathrm{FB}}$, one can place all of the uncertainty about the ratio of these responses into $\mathrm{C}^{\mathrm{FB}}$. Due to low tolerances and stray capacitance, this component value is uncertain, and needs to be determined from a calibration.

This calibration is done with a laser diode source with special drive circuitry. The diode (Toshiba T0LD9221M, $670 \mathrm{~nm}$ ) can be driven in two modes, selectable with a switch or a computer command. In mode 1, the pulse shape mimics that of the Nd:YAG laser with a width of $8 \mathrm{nsec}$, and in this mode the repetition rate is $250 \mathrm{kHz}$. In mode 2, the diode is pulsed with a square wave with an on time of $8 \mu$ s and a repetition rate of $250 \mathrm{~Hz}$. In this mode, the pulse width is long enough to be equivalent to DC for the slow circuit. The light output from the diode is monitored by a photodiode with a long integration time $(\sim 20 \mathrm{~ms})$. Since the duty cycle $(\mathrm{D}=$ $1 / 500)$ is the same in the two modes, the photodiode monitor readings are also roughly equal. An APD is illuminated with a fiber optic from this laser diode source through the alternate 
illumination path mentioned in Section II. The laser diode drive circuitry also sends a trigger to a dedicated input on the synchronizer module in the SHMUX nest for appropriate timing. The fast output voltage measured during mode 1 operation is then compared to the slow output for mode 2 operation. From equations 1 and 2, we write:

$$
\frac{V^{\text {slow }}}{V^{\text {fast }}}=\frac{G^{\text {slow }}}{G^{\text {fast }}} R^{F B} C^{F B} \frac{I_{p h}^{\text {slow }}}{N_{p h}^{\text {fast }}}=\frac{G^{\text {slow }}}{G^{\text {fast }}} \frac{R^{F B} C^{F B}}{\tau}
$$

and $\quad \boldsymbol{\tau}=\frac{P_{1}}{P_{2}} \frac{D}{f^{f a s t}}$

where $\mathrm{P}_{1}$ and $\mathrm{P}_{2}$ are the photodiode readings in the two modes, $\mathrm{D}=1 / 500$ is the duty cycle, and $\mathrm{f}^{\text {fast }}=2.5 \times 10^{5} \mathrm{~Hz}$ is the repetition rate in mode $1 . \tau$ is the effective square wave width of the mode 1 pulse. In practice, we measure the ratio in equation 3 to be $30-50 \%$ higher than the predictions from the nominal values, indicating a higher effective value of $\mathrm{C}^{\mathrm{FB}}$. Determining this value for each APD-preamplifier combination allows one to accurately relate pulsed and DC measurements.

\section{PERFORMANCE}

The linearity of the fast output was checked by imaging $8 \mathrm{nsec}$ pulses of light from the laser diode from one optical fiber, through a stack of identical neutral density filters, to another fiber connected to the alternate illumination port. Changing the number of filters varied the illumination over a range of 150 . At each illumination level, 300 samples were analyzed for the average and RMS noise. The response was linear to better than $1 \%$.

To analyze the noise data, it is useful to convert the linearity measurements to units of photoelectrons. To determine $\mathrm{M}$ as a function of bias voltage, a stable DC light source is used to illuminate the APD. At low bias voltage $\left(\mathrm{V}_{\text {bias }} \sim 25 \mathrm{v}\right)$, the $\mathrm{APD}$ functions as a unity gain photodiode. Recording the response in the slow channel, while scanning the bias voltage, and 
taking the ratio to the response at $25 \mathrm{v}$ yields $\mathrm{M}\left(\mathrm{V}_{\text {bias }}\right)$. Knowing $\mathrm{M}$ and $\mathrm{C}^{\mathrm{fb}}$ for the detector and preamplifier, one can convert the fast voltage response in the linearity scan to a photoelectron response, which can be used to measure various noise sources. Figure 4 shows the resulting dependence of the measured RMS photoelectrons $\left(\sigma_{\mathrm{f}}\right)$ on the measured photoelectron input $(\mathrm{S})$. This dependence was fitted to the following model:

$$
\sigma_{\mathrm{f} 0}=28.3 \pm 1.7 \text { photoelectrons }
$$

$\sigma_{f}=\left(\sigma_{f 0}^{2}+F S+L^{2} S^{2}\right)^{1 / 2}$

with fitted values:

$$
\begin{aligned}
& \mathrm{F}=2.32 \pm 0.08 \\
& \mathrm{~L}=.0096 \pm .0001
\end{aligned}
$$

where $\sigma_{\mathrm{f} 0}$ is the readout noise in the fast output in the absence of light input, $\mathrm{F}$ is the excess noise of the APD (see ref. 7), and L is the fractional pulse-to-pulse source variation in the laser diode. Measurements of $\sigma_{\mathrm{f} 0}$ have been carried out with no illumination on a number of the APDpreamp combinations with measured values ranging from 20 to 30 at $\mathrm{M}=75$.

The RMS noise in the fast channel was also measured using a stable DC light input with no pulsed input, to characterize the noise rejection for plasma background light. Converting to units of photoelectrons at $\mathrm{M}=75$, the RMS noise in the fast output was $\sigma_{\mathrm{f}}=104$ photoelectrons for an input of $\mathrm{I}_{\mathrm{S}}=1.05 \times 10^{11}$ photoelectrons/s. One can derive from these measurements an equivalent noise gate for white noise:

$$
t_{g}=\frac{\sigma_{p}^{2}}{F I_{s}}=45 n s
$$

As an experimental check to see if the $100 \mathrm{nsec}$ group delay was optimum for the Bessel filter, a similar measurement was done with the Bessel filter configured for a 50 nsec group delay. In this configuration we found a $15 \%$ reduction in the noise in the pulse channel, reflecting the expected narrowing of the noise bandwidth. There was no observable drop in the fast channel response, as 
shown in Fig. 2, indicating that a shorter group delay would be a modest improvement, worth implementing on the next batch of preamplifiers.

\section{SUMMARY}

An APD electronic readout system has been installed and tested that provide high sensitivity and good noise rejection for Thomson scattering measurements. The system includes a number of features that facilitate accurate readout and flexibility for various types of calibrations, including one that relates sensitivity for pulsed light inputs to DC inputs. Similar to previous designs, the system utilizes dual output preamplifier modules with fast and slow outputs. Individual programmable bias supplies are included in the preamplifier module, which are programmed to compensate for thermal drift. An autozero circuit eliminates DC offset drift in the slow channel. Outputs from the preamplifiers from a single polychromator are sampled and multiplexed by a SHMUX module for input to a general purpose 12 bit digitizer. Programmable delays are included to facilitate computer controlled synchronization.

\section{Acknowledgements}

The authors wish to thank J. Lowrance at PSI for help in the design, G. Gibilisco and J. Carson at PPPL for their fabrication effort, and P. Roney at PPPL and D. LeFrance at Drexel for their help in testing of this detector system. This work was performed under the auspices of the U.S. Department of Energy by Princeton Plasma Physics Laboratory under Contract DE-AC02-76CHO3073.

\section{References}

${ }^{1}$ H. Rohr, K. -H. Steuer, H. Murmann, and D. Meisel, IPP Report III/121B, July, 1987.

${ }^{2}$ H. Murmann, et al, Rev. Sci. Instrum. 63, 4941 (1992)

${ }^{3}$ T. N. Carlstrom, et al, Rev. Sci. Instrum. 63, 4901 (1992) 
${ }^{4}$ P. Pizzolati, et al, Rev. Sci. Instrum. 63, 4403 (1992)

${ }^{5}$ K. Narihara, et al, Rev. Sci. Instrum. 65, 4607 (1995)

${ }^{6}$ D. Johnson, et al., Rev. Sci. Instrum., 70, 776 (1990)

${ }^{7}$ P. P. Web, R. J. McIntyre, and J. Conradi, RCA Review 35, 234 (1974)

${ }^{8}$ C. L. Hsieh, et al., Rev. Sci. Instrum., 61, 2855 (1990)

${ }^{9}$ A. Bregni and L. Giudicotti, J. Phys. E: Sci. Instrum. 15 (1982)

\section{Figure Captions}

Fig. 1. Circuit schematic for the APD preamplifier module.

Fig. 2. Waveform showing the response of the pulse output of the preamplifier module to an 8 nsec laser diode pulse for a Bessel filter with a $100 \mathrm{nsec}$ group delay (black) and a 50 nsec group delay (gray).

Fig. 3. Photograph showing a filter polychromators (f), a preamplifer module (p), a SHMUX module (m), and a synchronization module (s).

Fig. 4. Variation of the RMS noise in the digitized fast output expressed in photoelectrons with the number of photoelectrons in the 8 nsec pulse at an APD gain of $M=75$. 


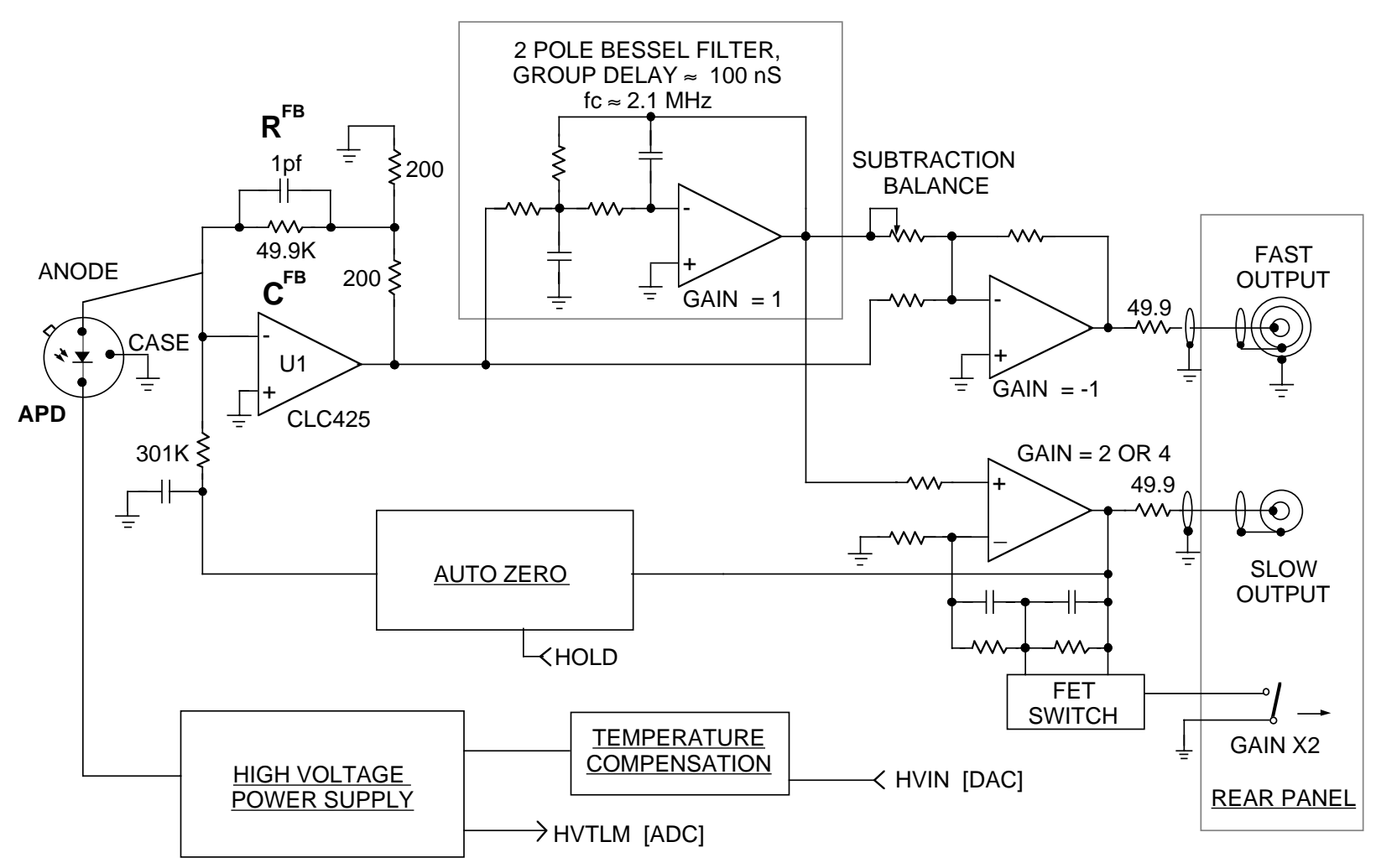

Figure 1. Circuit schematic for the APD preamplifier module. 


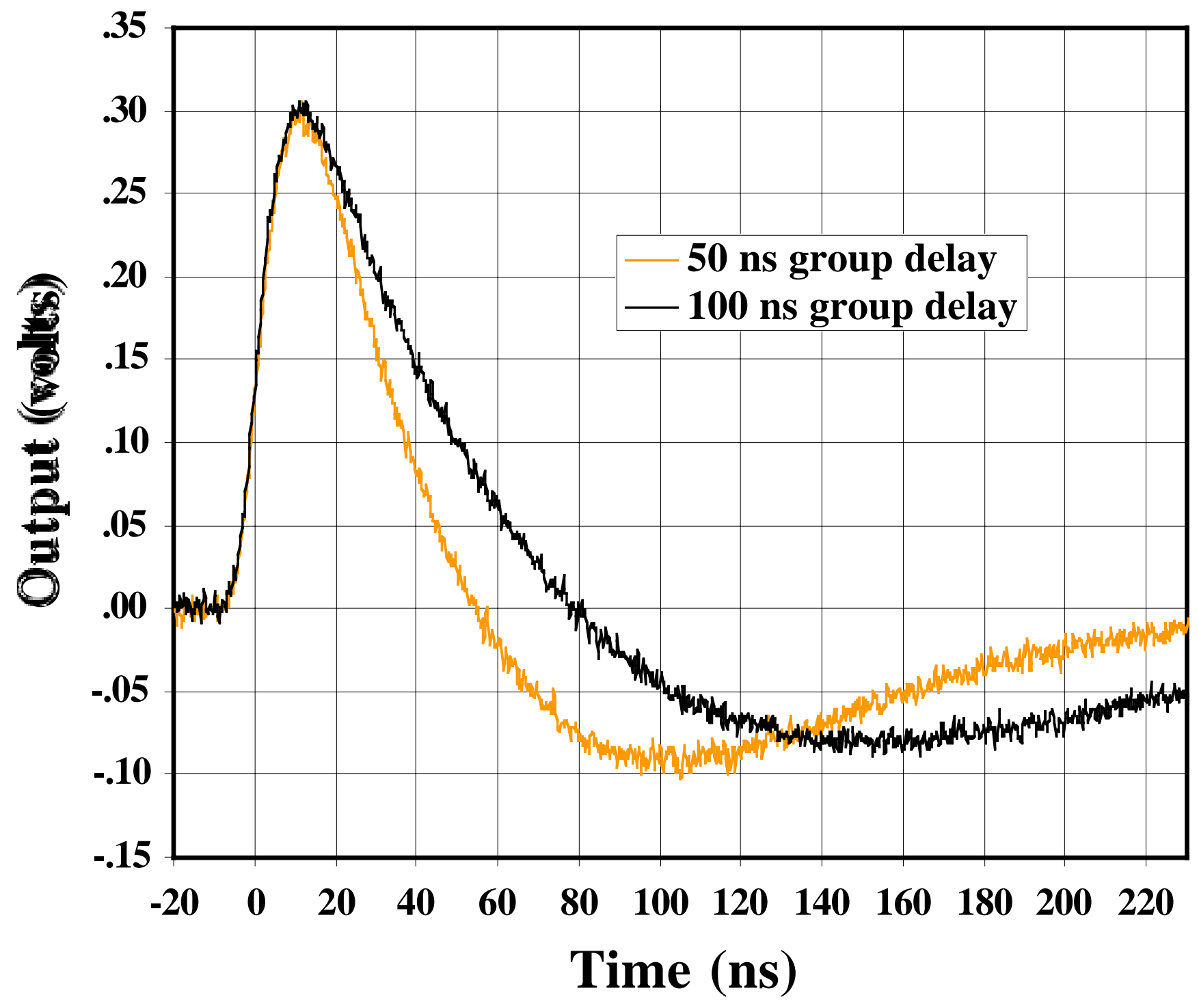

Figure 2. Waveform showing the response of the pulse output of the preamplifier module to an 8 nsec laser diode pulse for a Bessel filter with a 100 nsec group delay (black) and a 50 nsec group delay (gray). 


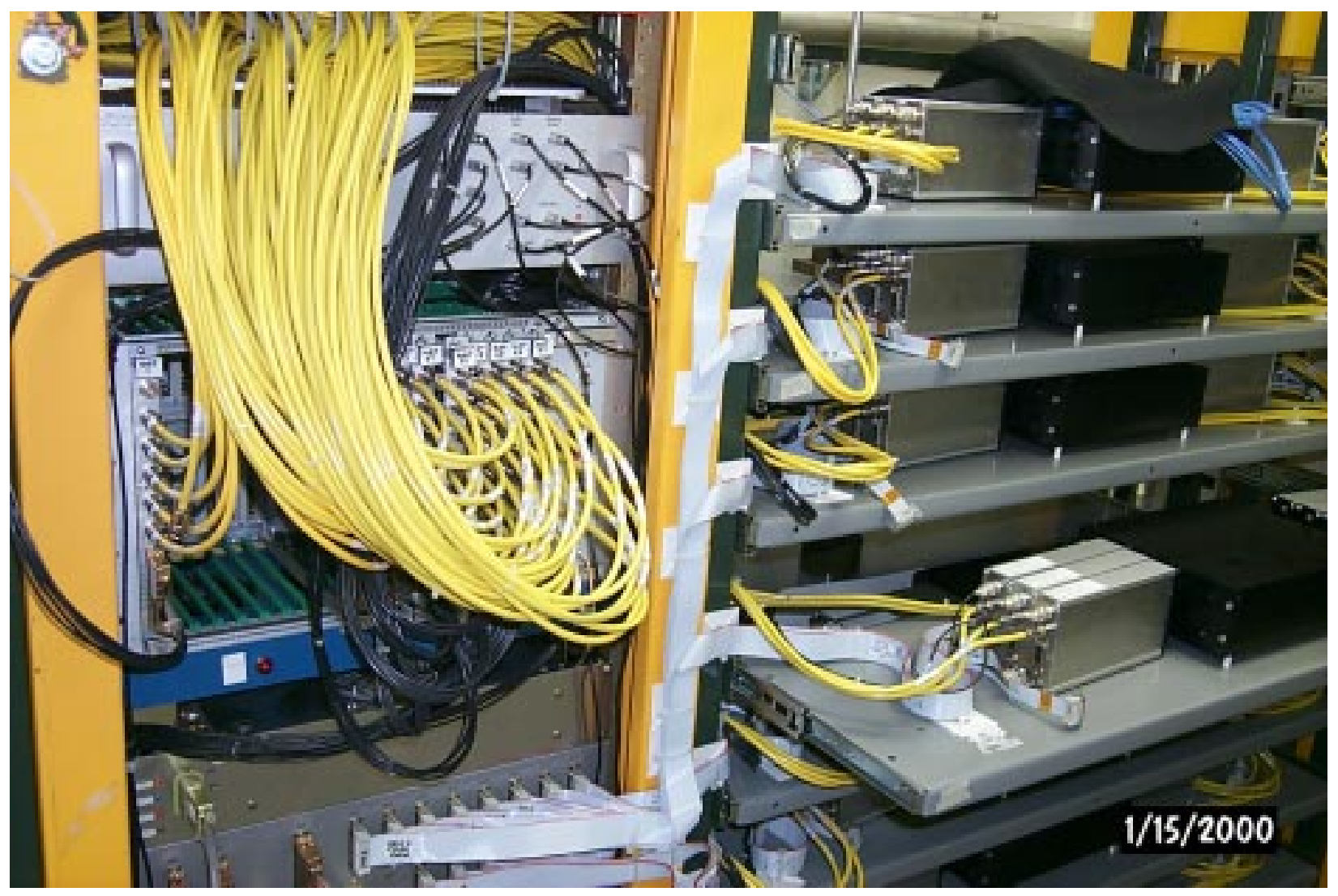

Figure 3. Photograph showing a filter polychromators (f), a preamplifer module (p), a SHMUX module $(\mathrm{m})$, and a synchronization module (s). 


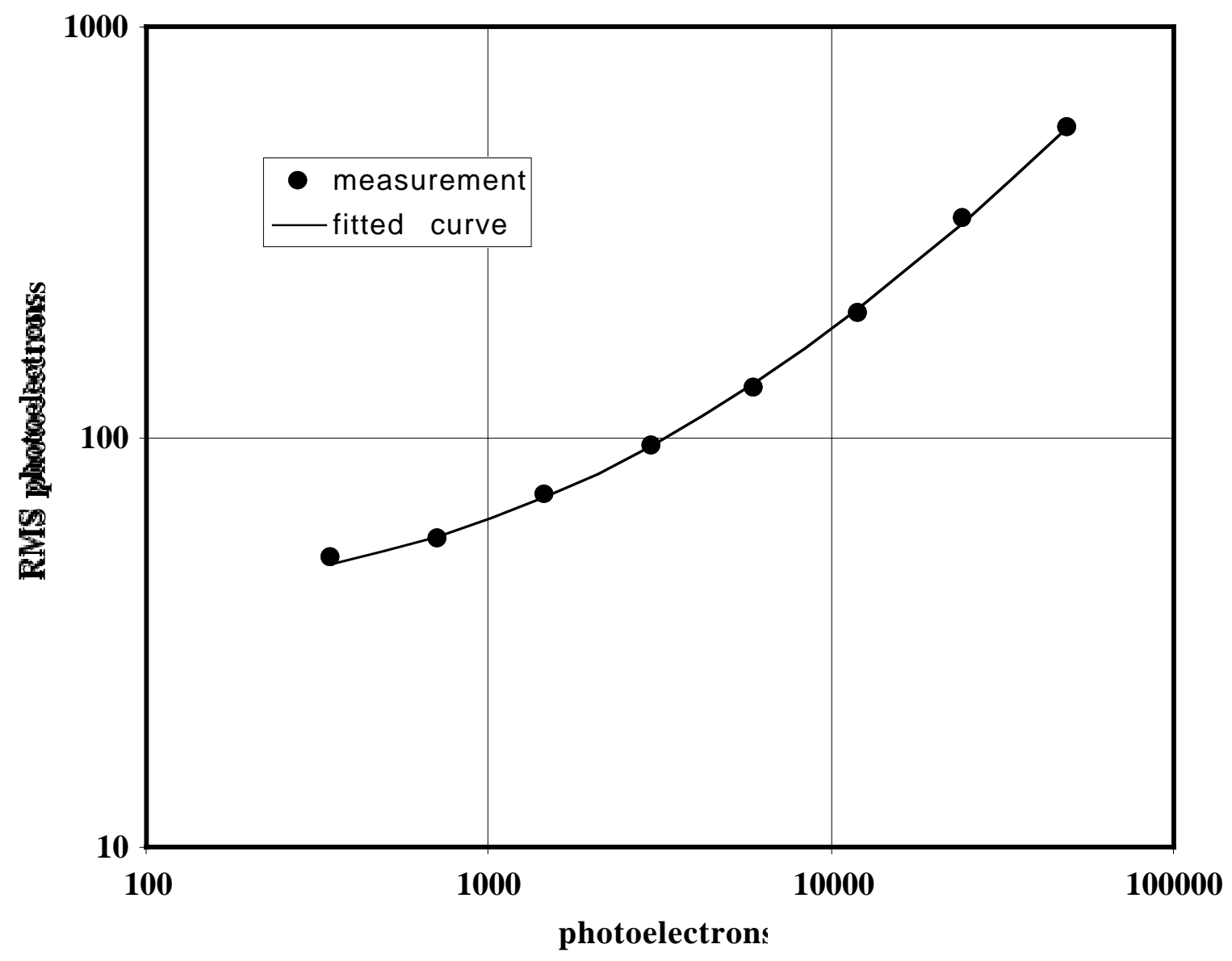

Figure 4. Variation of the RMS noise in the digitized fast output expressed in photoelectrons with the number of photoelectrons in the 8 nsec pulse at an APD gain of $\mathrm{M}=75$. 
The Princeton Plasma Physics Laboratory is operated by Princeton University under contract with the U.S. Department of Energy.

\author{
Information Services \\ Princeton Plasma Physics Laboratory \\ P.O. Box 451 \\ Princeton, NJ 08543
}

Phone: 609-243-2750

Fax: 609-243-2751

e-mail: pppl_info@pppl.gov

Internet Address: http://www.pppl.gov 